\title{
The CMB as a dark energy probe
}

\section{Carlo Baccigalupi*}

ITA, Albert Überle Strasse 2, 69120 Heidelberg, Germany, and SISSA/ISAS, Via Beirut 4, 34014 Trieste, Italy

E-mail: baccieita.uni-heidelberg.de, bacciesissa.it

\section{Viviana Acquaviva}

SISSA/ISAS, Via Beirut 4, 34014 Trieste, Italy

E-mail: acqua@sissa.it

\begin{abstract}
We give a brief review of the known effects of a dynamical vacuum cosmological component, the dark energy, on the anisotropies of the cosmic microwave background (CMB). We distinguish between a "classic" class of observables, used so far to constrain the average of the dark energy abundance in the redshift interval in which it is relevant for acceleration, and a "modern" class, aiming at the measurement of its differential redshift behavior.

We show that the gravitationally lensed CMB belongs to the second class, as it can give a measure of the dark energy abundance at the time of equality with matter, occurring at about redshift 0.5 . Indeed, the dark energy abundance at that epoch influences directly the lensing strength, which is injected at about the same time, if the source is the CMB. We illustrate this effect focusing on the curl (BB) component of CMB polarization, which is dominated by lensing on arcminute angular scales. An increasing dark energy abundance at the time of equality with matter, parameterized by a rising first order redshift derivative of its equation of state today, makes the BB power dropping with respect to a pure $\Lambda \mathrm{CDM}$ cosmology, keeping the other cosmological parameters and primordial amplitude fixed. We briefly comment on the forthcoming probes which might measure the lensing power on CMB.
\end{abstract}

$C M B$ and Physics of the Early Universe

20-22 April 2006

Ischia, Italy

\footnotetext{
${ }^{*}$ Speaker.
} 


\section{Fighting against a Cosmological Constant}

There are conceptually two ways in which the vacuum energy manifested in the Einstein equations:

$$
G_{\mu v}+\Lambda g_{\mu v}=8 \pi G T_{\mu \nu}+V g_{\mu v} .
$$

The first one, represented by $\Lambda$ above, has a pure geometrical conception and was introduced by Einstein himself for reconciling a static cosmology with general relativity, a mistake marked by himself as his biggest blunder. The second one, represented by $V$ in the equation above, was reintroduced afterwards by quantum mechanical arguments.

There is basically no expectation for $\Lambda$, while $V$ might be of the order of the energy scale at which all forces are predicted to unify by quantum gravity arguments, the Planck energy density $\rho_{\text {Planck }}$, simply because that is expected to be a fundamental scale, and quantum mechanics does not protect the vacuum from possessing a non-zero energy density. The Cosmological Constant Problem (CCP) comes from the fact that the vacuum energy is not compatible with the cosmological picture we guess, as it causes an exponential expansion which prevents structures to grow; this implies that the two vacuum energy terms above have to cancel out with a fantastic precision, leaving a residual which must be comparable or smaller than the present energy density; the latter is about 123 orders of magnitude lower than $\rho_{\text {Planck }}$, which implies

$$
\frac{|\Lambda-V|}{\rho_{\text {Planck }}} \lesssim 10^{-123}
$$

The CCP problem simply points out that there is at the present no explanation why the number above is so small, regardless that it is exactly zero or not. If one believes to the recent data from Type Ia Supernovae (SNIa, [1, 2]), Cosmic Microwave Background (CMB) and Large Scale Structure (LSS) combined (see [3] and references therein), giving evidence for acceleration in the cosmic expansion, the vacuum energy density today is actually non-zero, being about $75 \%$ of the critical one, with a few percent precision. This means that the number in the equation above is non-zero, of the order of $10^{-123}$ with percent precision. Technically speaking, this evidence is not representing a new problem with respect to the CCP, but rather it is renewing the interest for it. The simplest explanation of the cosmic acceleration in terms of a Cosmological Constant immediately raises two problems, known as fine tuning and coincidence, respectively. Why the vacuum energy is so small with respect to the typical values in the early universe? Why is it comparable with the matter energy density at the present? In response to this embarrassment, cosmologists created a broader concept of cosmological vacuum component, the dark energy, which may be dynamic and reduces to the Cosmological Constant in the static limit $[4,5]$. The measure with the highest possible accuracy of how much the dark energy is close to a Cosmological Constant is likely to be one of the major challenges for cosmology in the forthcoming decades. In this work we make a brief review of the dark energy effects on the CMB anisotropies. In section 2 we describe how the dark energy is usually parameterized. In section 3 we review how the CMB has been used so far to constrain the dark energy. In section 4 we list the new ideas of using the CMB as a dark energy probe, focusing on the gravitational lensing effect. In section 5 we show how the lensed CMB polarization, and its curl component in particular, depends on the dark energy abundance at the onset of acceleration. Finally, in section 6 we briefly comment on the forthcoming CMB experimental probes. 


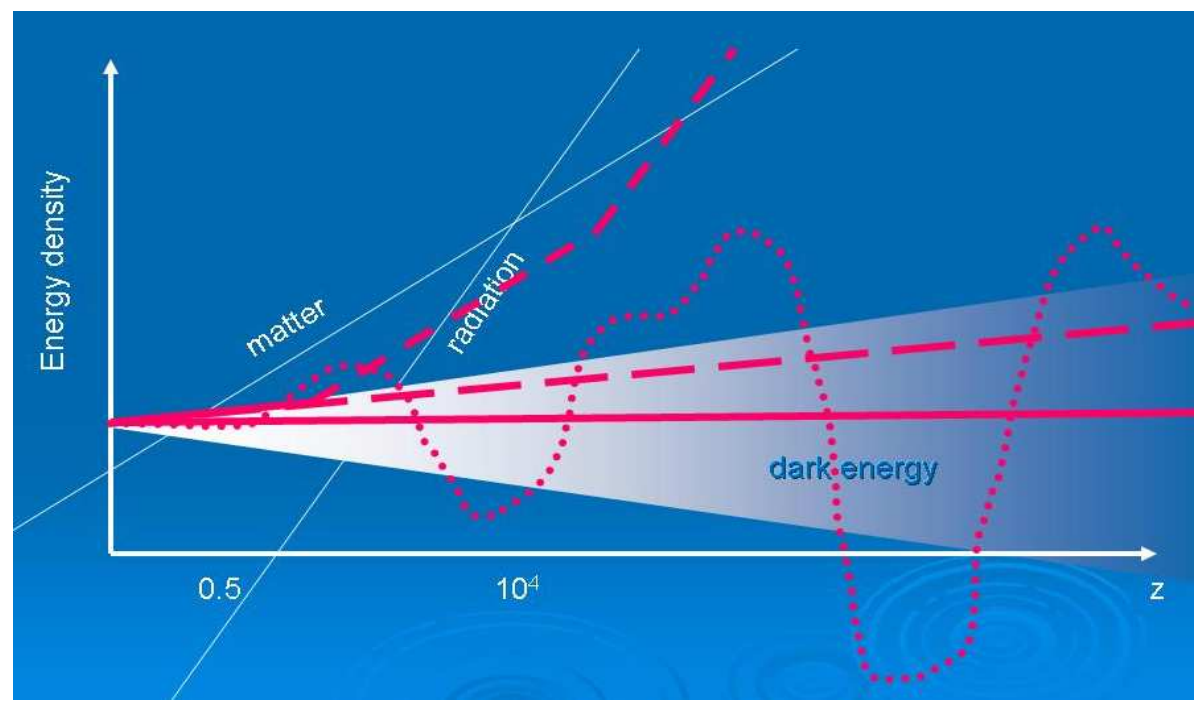

Figure 1: A representation of the behavior of non-relativistic, relativistic matter and different models of dark energy, as a function of the redshift on a logarithmic scale.

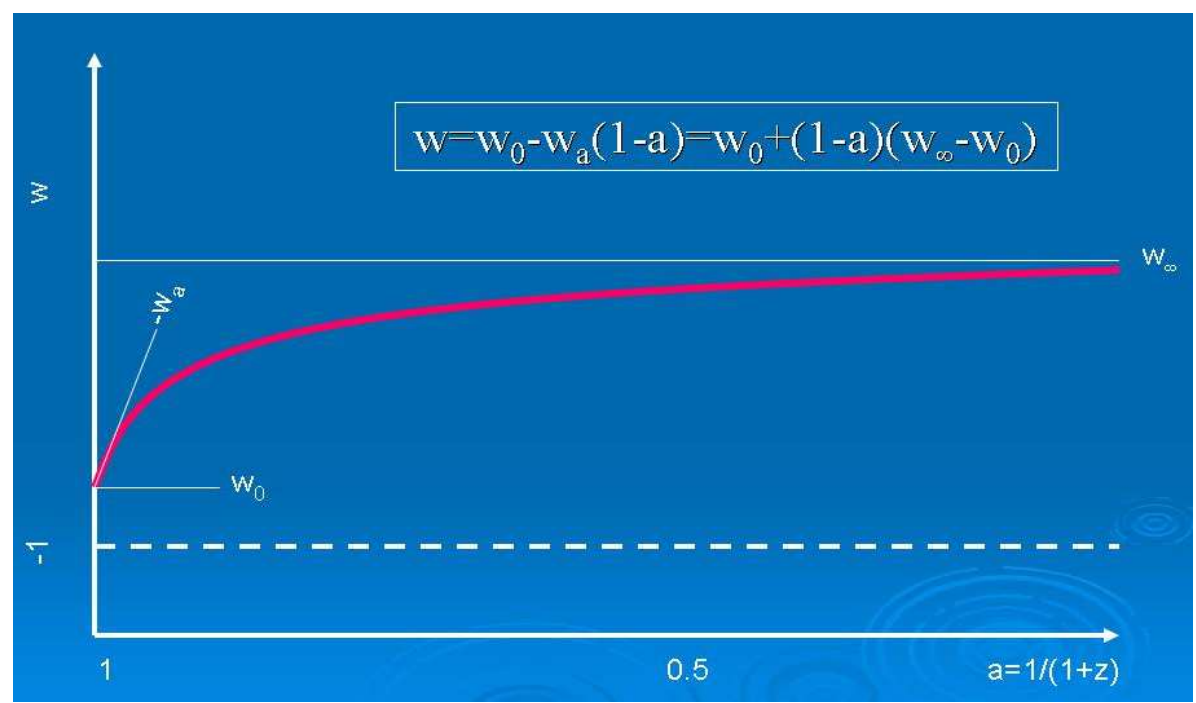

Figure 2: The behavior of the dark energy equation of state when its value and first derivative at present are given.

\section{Parameterizing cosmic acceleration}

The main dark energy unknown is its abundance as a function of the redshift, $\rho(z)$. That is conveniently parameterized in terms of the ratio between pressure and energy density, the equation of state $w(z)$; the latter fixes $\rho$ by means of the Einstein conservation equation, which implies

$$
\rho=\rho_{0} \exp \left[3 \int_{0}^{z} \frac{(1+w) d z^{\prime}}{1+z^{\prime}}\right]
$$

where $\rho_{0}$ represents the present dark energy density. In figure 1 we sketch the possible behaviors that the dark energy might have, and that have been proposed in the literature. The straight lines 
represents the scaling of non-relativistic and relativistic matter, as $(1+z)^{3}$ and $(1+z)^{4}$, respectively. The shaded area represents our ignorance on dark energy, namely the fact that we know with percent accuracy its abundance today (see [3] and references therein) while the uncertainty increases fast with the redshift. Together with the Cosmological Constant behavior, the dark energy might follow the dominant component (early quintessence, short dashed line, [5]), have an almost constant equation of state but larger than -1 (tracking quintessence, dashed line, [4]), or have some other complicated behavior, represented with dots in the figure; for a more complete review of the different models, see [6]. In the first two cases, the dark energy trajectories represent attractors which may solve, at least classically, the first of the two problems affecting the Cosmological Constant mentioned in the previous section.

If one has to measure the redshift function $\rho(z)$, the most unbiased approach would be to bin the redshift interval in which the dark energy is relevant, and provide some measure of $\rho$ in each bin. This approach is model independent by definition, has been already considered in the literature (see e.g. $[7,8]$ ), and will eventually be the standard with the increase in accuracy of the cosmological experiments. On the other hand, it has the disadvantage to increase the number of quantities to measure, with consequent decrease in accuracy until the data will have the appropriate quality. A viable alternative is represented by the parameterization of the few fundamental dynamical quantities that the dark energy has in any model. A convenient choice $[9,10]$ is represented by the present value of the equation of state, $w_{0}$ and its first derivative in the scale factor $w_{a}$; the latter simply measures the difference between $w_{0}$ and the asymptotic value in redshift, $w_{\infty}$ :

$$
w=w_{0}-w_{a}(1-a)=w_{0}+(1-a)\left(w_{\infty}-w_{0}\right) .
$$

The behavior of this function is shown in figure 2. Although its appearance is markedly different from the existing models, represented in figure 1, it catches the relevant behavior of $w$ at least close to the present, and that's the reason of its wide use.

\section{3. "Classic" dark energy effects on CMB and their role in the current constraints}

The dark energy modifies the cosmic expansion in the epoch in which it is relevant, i.e. at $z \lesssim 0.5$. As it is easy to see from (2.1), w>-1 means higher density, and consequently an higher Hubble parameter $H$, at all epochs starting from the same value today. The CMB effects induced by this modification were historically the first ones to be exploited to constrain the dark energy, and they are "classic" in this sense.

The first one is known as projection effect, and is merely due to the change in the distance of the last scattering surface induced by the modified expansion history. The distance to the last scattering $(l s)$ is easily evaluated as

$$
D=c H_{0}^{-1} \int_{0}^{z_{l s}} \frac{d z}{\sqrt{\sum_{i} \rho_{i} / \rho_{c 0}}}
$$

where $c$ is the light velocity, $H_{0}$ the Hubble expansion rate today, $\rho_{c 0}$ is the value of the cosmological critical density today, and the index $i$ runs over all cosmological components. Following the example above, $w>-1$ means that the last scattering surface gets closer, making all features in the CMB anisotropy appearing on larger angular scales. The mechanics of this effect is represented in figure 3; the reported formula is a simplification of (3.1) when all components have a constant 


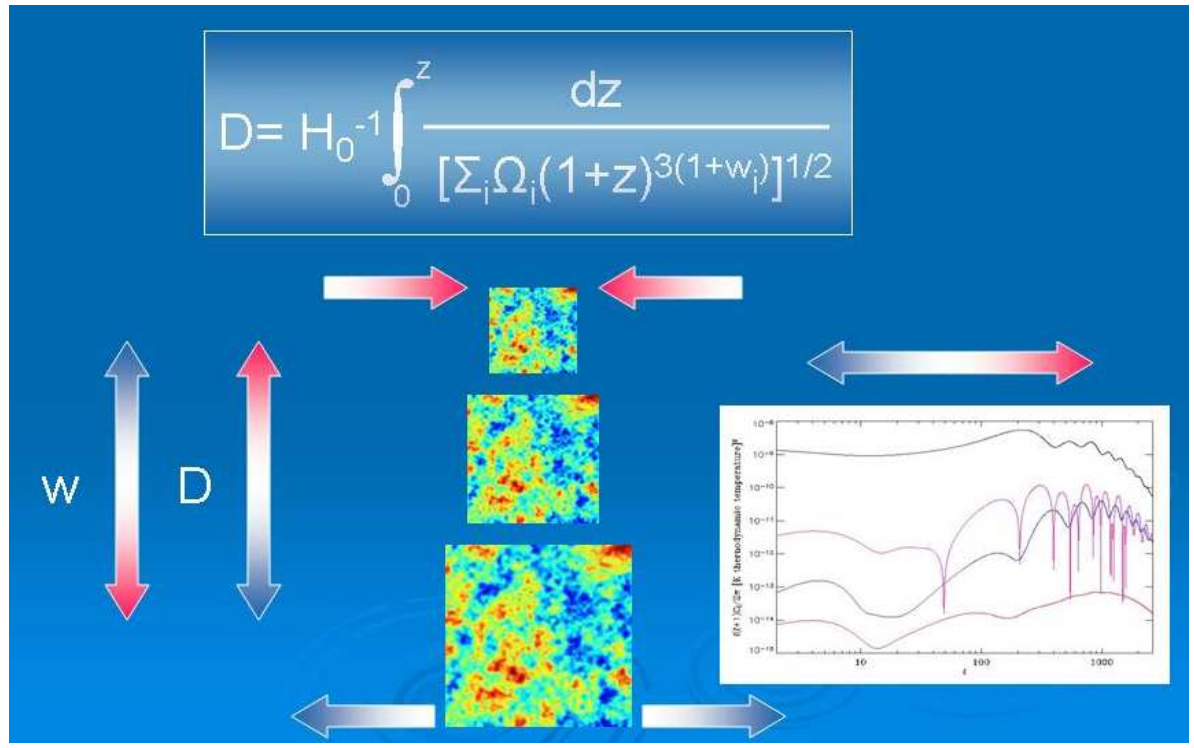

Figure 3: The dark energy dynamics alter the distance to last scattering, causing the same features of CMB anisotropies appearing on different angular scales.

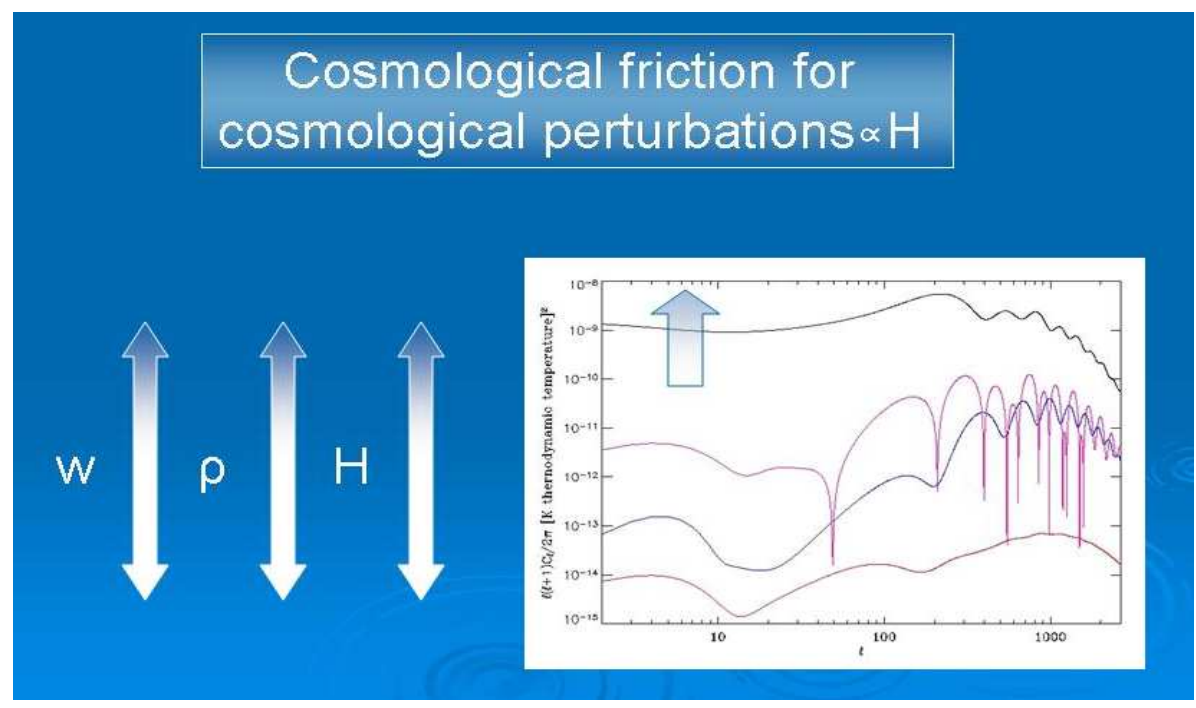

Figure 4: The variation of $H$ induced by the dark energy dynamics boosts anisotropies on large angular scales.

equation of state, and defining the parameters $\Omega_{i}=\rho_{i 0} / \rho_{c 0}$. In the bottom right panel the angular power spectrum of CMB anisotropies is shown for total intensity (TT, top curve), cross correlation between total intensity and polarization (TE, second curve from above), gradient and curl components of $\mathrm{CMB}$ polarization (EE and $\mathrm{BB}$, third and fourth curves from above, respectively); the whole spectra shift to the right or left under the effect of dark energy, as the multiple $l$ parameterizes the inverse of the angle at which the anisotropy power is measured.

The second effect comes from the different behavior of cosmological perturbations induced by the modified expansion rate, and is known as Integrated Sachs-Wolfe effect (ISW). An higher $H$ 
makes an higher cosmological friction, enhancing the dynamics that hills and wells in the gravitational potential undergo between last scattering and the present. As photons cross them, TT $\mathrm{CMB}$ anisotropies are boosted, but only on large angular scales, since on smaller ones the effect is washed out by the superposition of many structures along the line of sight. The effect is purely metric, and does not affect polarization as it does not involve electromagnetic scattering of photons. It is illustrated in figure 4.

The two effects above are both caused by the effect of the dark energy abundance integrated over the redshift. In the case of the projection, the redshift dependence of the dark energy density is actually washed out by the two redshift integrals $(2.1,3.1)$. Clearly this prevents from measuring the dark energy abundance at different redshifts, and also introduces degeneracies with other parameters which may also modify the redshift averaged expansion history. Again following the example above, the reduction of the distance to last scattering due to a dark energy with $w>-1$ is also induced in closed cosmologies, where distances are contracted. Despite of these degeneracies, the CMB is a pillar of the present constraints on the dark energy: the combination of the data from LSS and CMB anisotropies represents a complementary probe of the expansion rate with respect to the SNIa; their combination allows to probe cosmologies where the dark energy equation of state is assumed to be a constant, indicating that in those scenarios the dark energy is a Cosmological Constant with roughly ten percent precision (see [3] and references therein for a more accurate quotation of best fit confidence region):

$$
w=w_{0}=-1 \pm 10 \% .
$$

Recently, an attempt has been made to measure the behavior of the dark energy at redshifts higher than one, exploiting the data from the Sloan Digital Sky Survey (SDSS) and Lyman- $\alpha$ forest; the results still indicate a Cosmological Constant within errors ([11], still we refer to the original paper for a precise quotation of errorbars):

$$
w_{z \simeq 0.3}=-1 \pm 10 \%, w_{z \simeq 1}=-1 \pm 25 \% .
$$

The effects we just described are largely used and known because they are common to all dark energy models. On the other hand there may be others arising in specific scenarios. For example, in early quintessence cosmologies where a non-vanishing dark energy density is present at last scattering, the latter process results affected, with consequent modification of the shape of the acoustic peaks [12].

\section{4. "Modern" CMB relevance for dark energy: the promise of lensing}

Despite of the remarkable experimental results exposed above, the theoretical difficulties related to the Cosmological Constant represent a motivation to push the battle a little forward. The entire redshift behavior of the dark energy has to be measured in the forthcoming years, at all epochs relevant for acceleration, going well beyond the present data on its redshift average. To this purpose, several probes are being studied in addition to the improvement of the data from SNIa, CMB and LSS, opening the way to the "modern" era of dark energy observations. In figure 5 we show a little scheme of the relevant cosmological epochs. The onset of acceleration is a recent process in terms of redshift, and overlaps with structure formation. For this reason most of the modern 


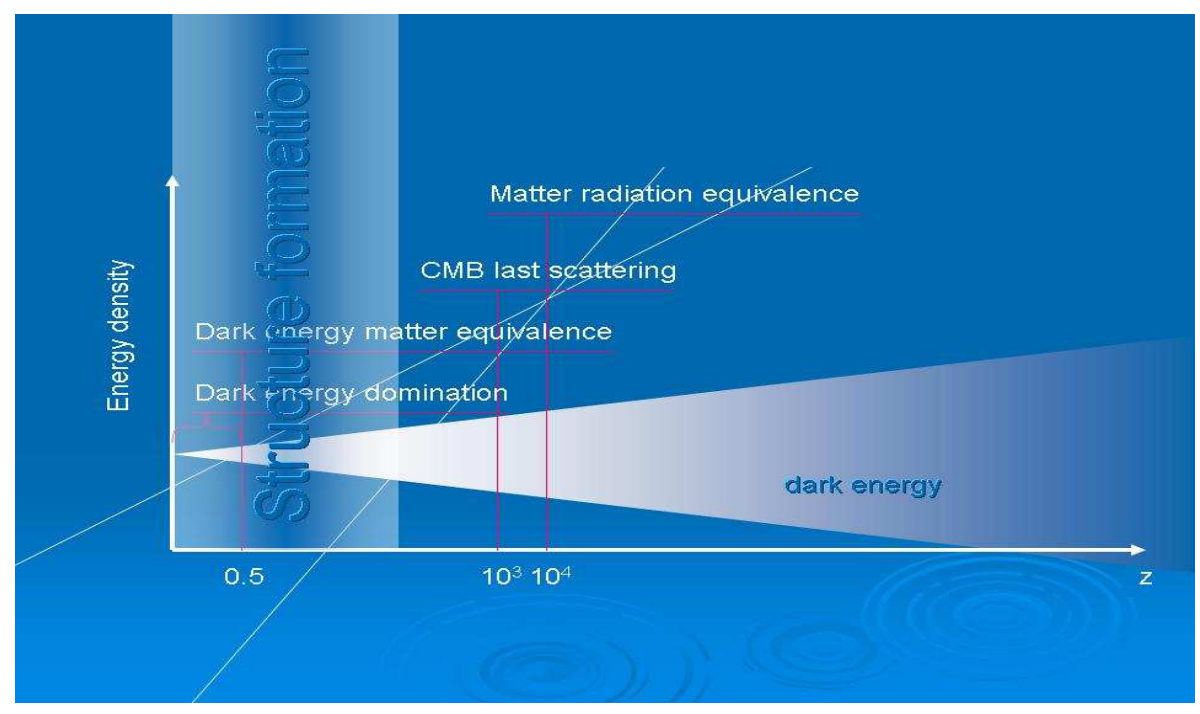

Figure 5: A representation of some relevant epochs in cosmology.

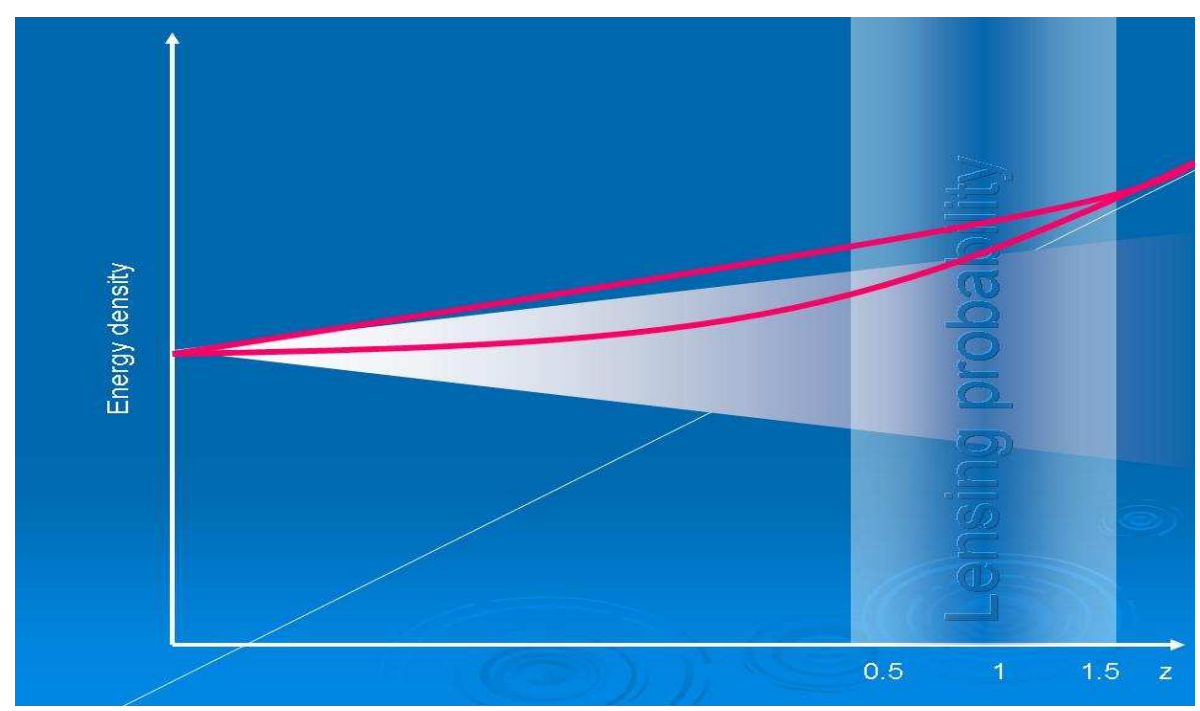

Figure 6: A representation of the lensing probability, distinguishing dark energy models at high redshifts.

dark energy probes try to exploit such overlap. Several authors are studying the dark energy relevance of effects like the oscillations imprinted by baryons in the dark matter distribution [13], the correlation between the ISW effect on the CMB and the observed LSS distribution [14], the gravitational lensing of galaxies [15] and CMB [16]; all these works are the latest ones of several by many authors which may be found in the references.

In this paper we make some comments on the last aspect only; the CMB lensing relevance for dark energy comes from an elementary geometric property of the gravitational lensing process: the lensing probability is zero if the lens position coincides with the observer or the source position. Therefore, any lensing observable picks up its signal roughly in the middle between source and observer. As sketched in figure 6, if the source is the CMB the lensing power peaks at about redshift 


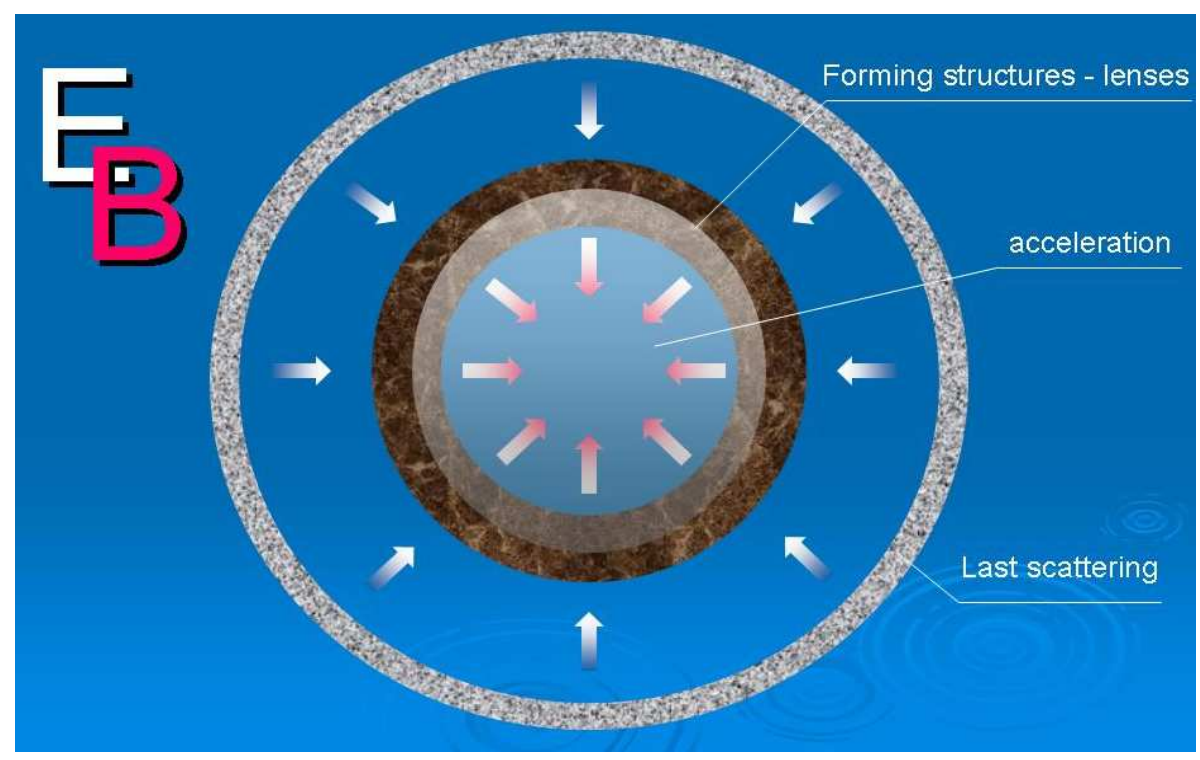

Figure 7: A sketch of the generation of lensing BB modes in the CMB polarization anisotropies.

1 (see [17] and references therein); this is very interesting for dark energy, because it means that the CMB lensing is potentially relevant for constraining its abundance at the onset of acceleration, independently on its present behavior.

The weak lensing of the CMB by large scale cosmological structures along the line of sight is a science per se, see e.g. [18] and references therein: it is relevant on the typical angular scales subtended by cosmological structures at $z \simeq 1$, say from a few arcminutes to the degree, and represents a second order cosmological effect, caused by perturbations in the matter density onto $\mathrm{CMB}$ anisotropies. Therefore it correlates different scales, making the overall CMB statistics nonGaussian, smearing out the acoustic peaks in the angular power spectrum, transferring power from the $\mathrm{EE}$ component of the $\mathrm{CMB}$ polarization to the $\mathrm{BB}$ one. This last effect is the one we focus on in the following.

\section{Lensing BB modes in CMB polarization}

The generation of lensing BB modes is described in figure 7. Even starting with a pure EE polarization at last scattering, a fraction of it becomes BB by lensing. Once again, this is due to the fact that CMB lensing is a second order effect for cosmological perturbations. Their independence at different wavelengths, valid at the linear level, no longer holds for lensing. The relevance of this effect on dark energy is also indicated in the figure, and is simply because the injection of $B B$ modes occurs at the onset of acceleration, and therefore should be sensitive to the dark energy abundance at that epoch. Another welcome feature is that the cosmological BB signal is expected to be dominated by lensing; any primordial power injected by gravitational waves peaks on the degree scale, and vanishes rapidly on smaller angles because of their relativistic behavior.

We now expose the relevant features of this argument, while a quantitative analysis can be found in [16]. Let us consider two different dark energy models, featuring the same equation of state today, but markedly different in the past, as in figure 8, top left panel; technically, those models 
correspond to a SUGRA ([19], dashed line) and inverse power law quintessence potentials ([4], solid line). They are suitable for our analysis, as they differ at high redshift. So we fix all cosmological parameters to be the same for the two models, including the primordial perturbation normalization, and we look at the lensed CMB angular power spectra in the top right and middle panels of figure 8. The plotted $C_{l} \mathrm{~s}$ are the coefficients of the expansion in Legendre polynomials of the CMB anisotropy two point correlation function in temperature and polarization, normalized to the average temperature in order to be dimensionless. While the TT and EE spectra undergo primarily a modest projection shift, the BB amplitude changes remarkably as a consequence of the modified value of $H$ at the epoch in which the lensing is injected, as a result of the different behavior in the two dark energy models. This is simply understood as follows: for a fixed primordial normalization, an higher value of $H$ results in an higher damping of perturbations because of cosmological friction, reducing the overall power of lensing. Indeed, the difference in amplitude of the BB modes traces the one in the value of $w$ at about redshift 1 ; for instance, the height of the lensing peak shows a $30 \%$ variation for the models in figure 8 , see the top left and middle right panel.

In order to demonstrate that this feature breaks the projection degeneracy mentioned above, in the two bottom panels of figure 8 we consider three different dark energy models with different values of $w_{0}$ and $w_{\infty}$ giving the same distance to last scattering (3.1); namely, the models have $w_{0}=$ $-0.8, w_{\infty}=-0.56$ (dotted line), $w_{0}=-0.9, w_{\infty}=-0.4$ (dashed line), $w_{0}=-0.965, w_{\infty}=-0.3$ (solid line). As it is evident, while the TT spectra are identical, the amplitude in BB changes remarkably.

As we stressed already, the quantitative assessment of how much this effect helps the determination of the redshift behavior from CMB lensing is ongoing [16]. On the other hand, the good amplitude of the effect, the enhanced sensitivity to the dark energy abundance at the onset of acceleration, independently on the present, and ultimately the easy explanation in terms of the known cosmological dynamics makes this feature interesting. If the amplitude of the lensing BB modes will ever be measured, it will be straightforward to check if the level is the same as predicted in a $\Lambda \mathrm{CDM}$ or different, as models with $w>-1$ at the onset of acceleration predict.

\section{Future CMB data and dark energy}

We conclude making some comments on the possibility to detect the lensing on the CMB in the near future. The attempts conducted so far on the data by the Wilkinson Microwave Anisotropy Probe (WMAP) were unsuccessful [20], while for the other experiments the lensing detection is hopeless due to instrumental limitations.

The forthcoming CMB probes are expected to have the instrumental capabilities to detect the lensing distortion in the $\mathrm{CMB}$ anisotropies, and in particular in the BB power in the polarization.

The Planck satellite (www.rssd.esa.int/Planck) has the appropriate sensitivity in order to detect the non-Gaussian power induced by lensing on TT anisotropies; as any lensing observable, such nonGaussian power is also injected at the onset of acceleration only. The potential relevance of this effect for constraining dark energy has also been addressed (see [21] and references therein).

The CMB polarization on degree and sub-degree angular scales, and the BB modes in particular, are the main targets of the next sub-orbital CMB probes, either ground based and balloon borne, in a 

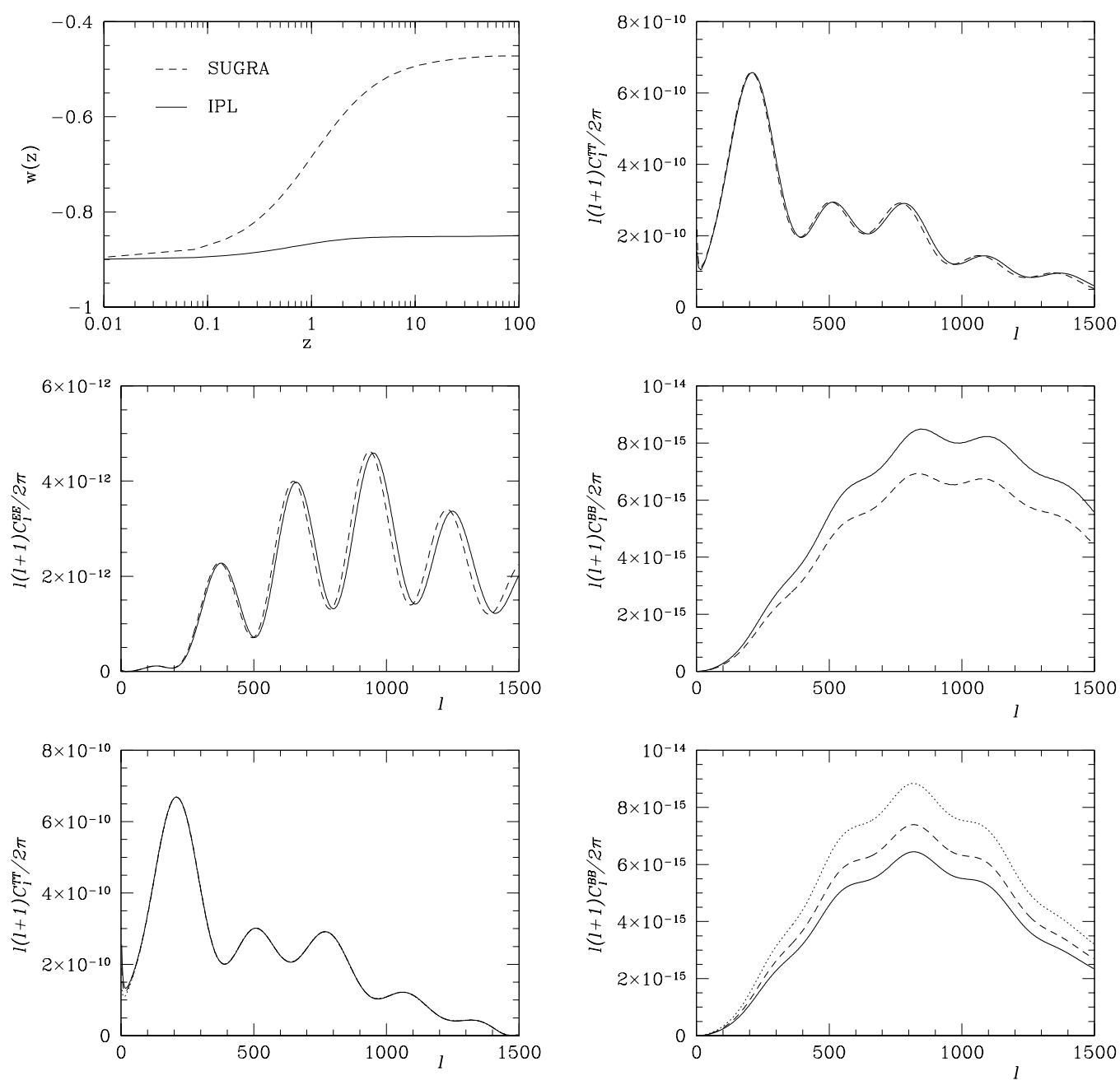

Figure 8: Lensed CMB spectra in dark energy cosmologies with different equation of state behavior (top left): TT spectra ( top right), EE spectra (middle left), BB spectra (middle right). Bottom: lensed TT (left) and BB (right) spectra for three dark energy models featuring the same distance from last scattering.

similar way as it was for the detection of the first peak in the CMB TT anisotropies, just a few years ago. There is no space to review all of them here, but a good list can be found at lambda.ngsc.gov where one can appreciate their number, and the consequent large theoretical and technological effort in this direction. As an example, in figure 9 we show the expected performance of the balloon borne $\mathrm{E}$ and B experiment (EBEx, see [22]). The error bars are calculated based on the expected detector sensitivity, number of detectors, sky coverage and integration time. They do not include calibration or other systematic uncertainties. The total BB signal, indicated as a solid line as the $\mathrm{EE}$ one, is made by a primordial contribution of tensor modes from the Inflationary Gravitational Background (IGB), plus the lensing power. As it can be seen, in principle that instrument should measure the level of the lensing BB power with good accuracy. For sure, such performance has to be revised in terms of systematics, but the figure also highlights one of the main obstacles to the observation of the cosmological $\mathrm{BB}$ power in the $\mathrm{CMB}$, which is represented by the diffuse fore- 


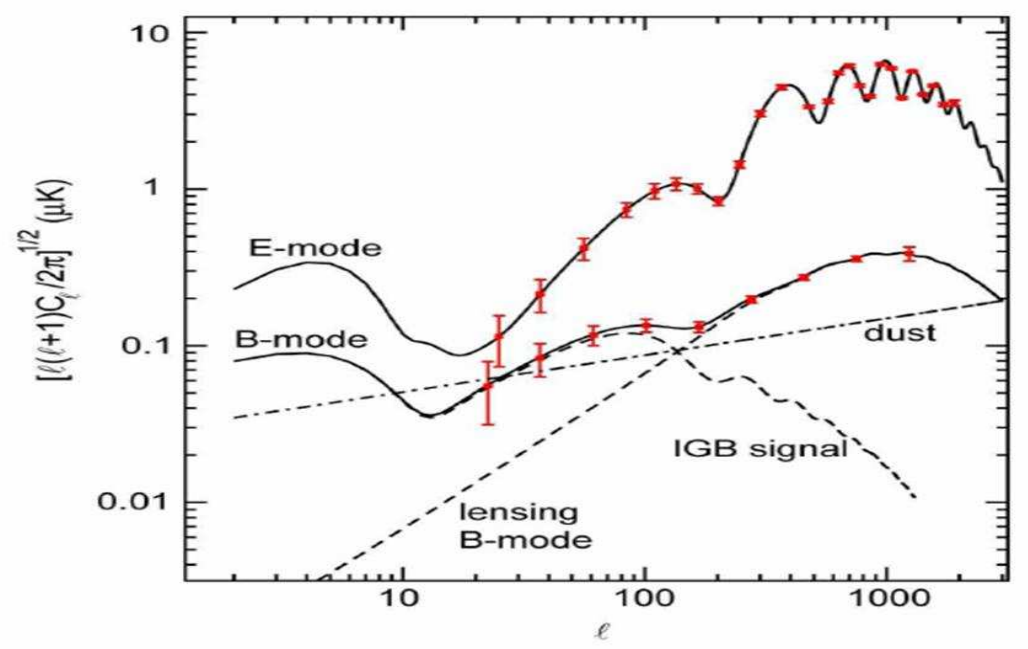

Figure 9: The expected performance of the EBEx experiment for CMB polarization measurements [22].

ground emission from our own Galaxy. At the frequencies of the EBEx experiment, between 150 and $450 \mathrm{GHz}$, the main contaminant is represented by the thermal dust emission, made by magnetized grains which get locally aligned by the Galactic magnetic field; its expectation at $150 \mathrm{GHz}$ is shown as a dashed line in the figure. While for CMB TT the sky is relatively free of foreground emission when the observation is targeted on a region far from the Galactic plane, for polarization, and $\mathrm{BB}$ modes in particular, the foreground contamination is potentially relevant on all sky regions, and at all frequencies, as the WMAP data recently confirmed [23]. On the other hand, the statistical difference between background and foregrounds might be exploited in order to reduce the foreground contamination in the forthcoming CMB experiments, see [24] and references therein.

In conclusion, we brought arguments here which show that the CMB has to be regarded as a probe of the differential behavior of the dark energy component, not only of its average as it has been so far. Its efficiency will be limited by instrumental systematics or foreground emission, but whether or not this will be worse than for other probes, e.g. supernovae or lensing of galaxies etc., will be clear only when the data will be actually taken.

\section{References}

[1] A.G. Riess et al., Observational Evidence from Supernovae for an Accelerating Universe and a Cosmological Constant, Astrophys. J. 116 (1998) 1009[astro-ph/9805201].

[2] S. Perlmutter et al., Measurements of Omega and Lambda from 42 High-Redshift Supernovae, Astrophys. J. 517 (1999) 565 [astro-ph/9812133].

[3] D.N. Spergel et al., Wilkinson Microwave Anisotropy Probe (WMAP) Three Year Results: Implications for Cosmology, submitted to Astrophys. J. (2006) [astro-ph/06034 49].

[4] B. Ratra, P.J.E. Peebles, Cosmological consequences of a rolling homogeneous scalar field, Phys. Rev. D 37 (1988) 3406.

[5] C. Wetterich, Cosmologies with variable Newton's “constant”, Nucl. Phys. B 302645 (1988). 
[6] P.J.E. Peebles, B. Ratra, The cosmological constant and dark energy, Rev. Mod. Phys. 75 (2003) 559 [astro-ph/0207347].

[7] R.G. Crittenden, L. Pogosian, Investigating dark energy experiments with principal components, (2005) [astro-ph/0510293].

[8] J. Dick, L. Knox, M. Chu, Reduction of Cosmological Data for the Detection of Time-varying Dark Energy Density, (2006) [astro-ph/0 603247].

[9] D. Chevallier, D. Polarski, Accelerating Universes with Scaling Dark Matter, International J. Mod. Phys. 10 (2001) 213 [gr-qc/ 0009008 ].

[10] E. V. Linder, Exploring the Expansion History of the Universe, Phys. Rev. Lett. 90 (2003) 091301 [astro-ph/0208512].

[11] U. Seljak et al., Cosmological parameter analysis including SDSS Ly $\alpha$ forest and galaxy bias: Constraints on the primordial spectrum of fluctuations, neutrino mass, and dark energy, Phys. Rev. D 10 (2005) 103515 [astro-ph/0407372].

[12] M. Doran, M. Lilley, J. Schwindt, C. Wetterich, Quintessence and the Separation of Cosmic Microwave Background Peaks, Astrophys. J. 559 (2001) 501 [astro-ph/0 012139].

[13] M. White, Baryon oscillations, Astropart. Phys. 24 (2005) 334 [a stro-ph/0 507307 ].

[14] L. Pogosian, P.S. Corasaniti, C. Stephan-Otto, R. Crittenden, R. Nicol, Tracking dark energy with the integrated Sachs-Wolfe effect: Short and long-term predictions, Phys. Rev. D 72 (2005) 103519 [astro-ph/0506396].

[15] W. H, B. Jain, Joint galaxy-lensing observables and the dark energy, Phys. Rev. D 70 (2004) 043009 [astro-ph/0312395].

[16] V. Acquaviva, C. Baccigalupi, Dark energy records in lensed cosmic microwave background, submitted to Phys. Rev. D (2005) [astro-ph/0507644].

[17] M. Bartelmann, P.Schneider, Weak gravitational lensing, Phys. Rep. 340 (2001) 291 [astro-ph/9912508].

[18] A. Lewis, A. Challinor Weak gravitational lensing of the CMB, Phys. Rep. 429 (2006) $1[$ astro-ph/0601594].

[19] P. Brax, J. Martin, Robustness of quintessence, Phys. Rev. D 62 (2000) 103502 [astro-ph/9912046].

[20] C.M. Hirata, N. Padmanabhan, U. Seljak, D. Schlegel, J. Brinkmann Cross-correlation of CMB with large-scale structure: Weak gravitational lensing, Phys. Rev. D 70 (2004) 103501 [astro-ph/0406004].

[21] F. Giovi, C. Baccigalupi, F. Perrotta, Cosmic microwave background constraints on dark energy dynamics: Analysis beyond the power spectrum, Phys. Rev. D 71 (2005) 103009 [103009].

[22] P. Oxley et al., The EBEX experiment, Earth Observing Systems IX. Edited by Barnes, William L.; Butler, James J. Proceedings of the SPIE 5543 (2004) 320 [astro-ph / 0501111 ].

[23] L. Page et al., Three Year Wilkinson Microwave Anisotropy Probe (WMAP) Observations: Polarization Analysis, submitted to Astrophys. J. (2006) [astro-ph/0603450].

[24] F. Stivoli, C. Baccigalupi, D. Maino, R. Stompor, Separating cosmological B modes from foregrounds in cosmic microwave background polarization observations, submitted to MNRAS (2005) [astro-ph/0505381]. 